This is the author's final, peer-reviewed manuscript as accepted for publication. The publisher-formatted version may be available through the publisher's web site or your institution's library.

\title{
Improving chemical production processes by selective byproduct removal in a pervaporation membrane reactor
}

Fan Zhang, Mary E. Rezac, Sudipto Majumdar, Praveen Kosaraju, Stuart Nemser

\section{How to cite this manuscript}

If you make reference to this version of the manuscript, use the following information:

Zhang, F., Rezac, M. E., Majumdar, S., Kosaraju, P., \& Nemser, S. (2014). Improving chemical production processes by selective byproduct removal in a pervaporation membrane reactor. Retrieved from http://krex.ksu.edu

\section{Published Version Information}

Citation: Zhang, F., Rezac, M. E., Majumdar, S., Kosaraju, P., \& Nemser, S. (2014). Improving chemical production processes by selective by-product removal in a pervaporation membrane reactor. Separation Science and Technology, 49(9), 12891297.

Copyright: Copyright @ Taylor \& Francis Group

Digital Object Identifier (DOI): doi:10.1080/01496395.2014.893444

Publisher's Link:

http://www.tandfonline.com/doi/full/10.1080/01496395.2014.893444\#.VDfjL8VdVQx

This item was retrieved from the K-State Research Exchange (K-REx), the institutional repository of Kansas State University. K-REx is available at http://krex.ksu.edu 


\title{
Improving Chemical Production Processes by Selective Byproduct Removal in a Pervaporation Membrane Reactor
}

\section{Running title: Selective Byproduct Removal in a Membrane Reactor}

\author{
Fan Zhang ${ }^{\mathrm{a}}$, Mary E. Rezac ${ }^{\mathrm{a}}$, Sudipto Majumdar ${ }^{\mathrm{b}}$, Praveen Kosaraju ${ }^{\mathrm{b}}$, Stuart Nemser ${ }^{\mathrm{b}}$ \\ ${ }^{a}$ Department of Chemical Engineering, Kansas State University, Manhattan, KS 66506-5102, USA \\ ${ }^{b}$ Compact Membrane Systems, Inc., 335 Water Street, Wilmington, DE 19804, USA
}

\begin{abstract}
Esters have applications as flavoring agents, pharmaceuticals, and cosmetics. Esterification and transesterification are convenient means to synthesize esters. Frequently, a large excess of one of the reactants is mandatory to obtain good yields for these equilibrium driven reactions. Selective removal of the reaction by-product allows for an increased yield. In this paper, the esterification of oleic acid with ethanol and the transesterification of methyl benzoate with n-butanol were studied in pervaporation membrane reactors equipped with perfluorinated composite membranes. A $95 \%$ yield of ethyl oleate was reached within 60 hours and the conversion of methyl benzoate increased from $52 \%$ to $77 \%$.
\end{abstract}

Keywords: Pervaporation; Composite membrane; Membrane reactor; Transesterification; Esterification 


\section{Introduction}

Membrane reactors have been investigated since the 1970s and are receiving increased attention as an alternative to distillation [1]. In a pervaporation-aided membrane reactor, a feed liquid mixture contacts one side of a membrane; one or more components of the liquid permeate through the membrane and is removed as a vapor from the other side. The partial pressure difference between the vapor pressure of the feed solution and the permeate promotes transport through the membrane [2].

Membrane reactors operating in pervaporation conditions have the potential to significantly increase product yields and reaction rates achieved in conventionally equilibrium-limited reactions. In a pervaporation-aided membrane reactor, since the membrane inside the reactor allows selective permeation of one product component (usually the by-product, such as water or low molecular weight alcohol) from the reaction mixture, it is possible to enhance the conversion of a thermodynamically or kinetically limited reaction by shifting the reaction towards higher yield direction[1, 3, 4]. As well as reaching higher yields, membrane reactors may also save energy and reduce cost $[2,5]$. A conventional reactor system requires a downstream separator to purify the products from any unreacted feeds and allow for recycle of unreacted feed. Conventional separation methods are often energetically demanding. Traditional removal technologies include distillation, extraction, adsorption, and crystallization. For compounds which are heat or solvent sensitive, chemical entrainers are necessary. In every case, the removal of byproducts requires large amounts of energy for direct heating, regeneration of any solvent or dehydrating agent, and treatment of the generated waste. Membrane reactors have the 
potential to simplify separations and reduce energy demand [6]. With a membrane reactor and its rapid selective removal of by-products, the separation of byproducts from the reaction mixture will be a smaller device, and the separation of the reaction products may no longer be necessary, thus saving energy and reducing cost.

Esters are very important fine chemicals. They have applications in a variety of areas such as cosmetics, flavors, pharmaceuticals, plasticizers, solvents, and monomers, and they can also serve conveniently as starting materials in multiple reactions. Esterification and transesterification reactions have been broadly employed in laboratory and industry for ester synthesis. Frequently, these reactions have low product yields due to the thermodynamic equilibrium limitation and a large excess of one of the reactants is mandatory to obtain good conversions. These reactions are well-suited for the use of a membrane reactor system in that if the by-product water or low molecular weight alcohol could be selectively removed from the reaction mixture, higher conversion than thermodynamic equilibrium conversion can be achieved and a large excess of one of the reactants may not be necessary.

Consequently, numerous studies have been conducted on ester synthesis via esterification reactions in membrane reactor systems [5, 7-14]. Recently, the esterification of oleic acid with ethanol has attracted research interest since this reaction is a typical model reaction widely studied as the pre-treatment step of lipids for the biodiesel (fatty acid esters) production [15-19]. Lipids used for biodiesel production usually contain more than $1 \%$ w/w free fatty acid (FFA) and water. FFA and water in the lipid feedstock can rapidly react with the base catalyst, consuming it and giving way to long chain soaps, which will 
reduce the yield and formation rate of the fatty acid esters, also bring difficulty for the separation of glycerol in the final step of the biodiesel production process [20, 21]. Thus a pre-treatment esterification step to reduce the FFA content in lipid feedstock to levels below $1 \% \mathrm{w} / \mathrm{w}$ must be considered as mandatory [22]. Lucena et al. used a reactor coupled with a zeolite 3A filled adsorption column to study oleic acid esterification with ethanol. An ethyl ester yield of $99.9 \pm 0.1 \%$ was obtained at $110^{\circ} \mathrm{C}$ with an initial ethanol to oleic acid molar ratio of 3 and $1 \% \mathrm{w} / \mathrm{w}$ sulfuric acid catalyst concentration [15]. This high conversion was achieved by using water removing zeolites which require ex-situ regeneration following use. While possible in the laboratory, such a technique is inappropriate for commercial-scale production. Rather, this work investigates the heterogeneously catalyzed esterification of oleic acid with ethanol to produce ethyl oleate in a membrane reactor system (Scheme 1).

Transesterification is an important synthetic process used as an alternative method to synthesize esters. On some occasions, transesterification is more advantageous than esterification. For instance, some carboxylic acids are sparingly soluble in organic solvents and according difficult to subject to homogeneous esterification. Transesterification is particularly useful when the parent carboxylic acids that can be used for esterification are labile or difficult to isolate [23]. While esterification reactions are the main class of reactions that have been studied in pervaporation membrane reactors, transesterification reactions have rarely been investigated in pervaporation membrane reactor systems because of the limited availability of pervaporation membranes that have long-term stability and sufficient selectivity of the product alcohol to the reactant alcohol [24-27]. This study is concerned with the homogenously catalyzed transesterification of 
methyl benzoate with butanol (Scheme 2) in an enhanced continuous pervaporation membrane reactor system.

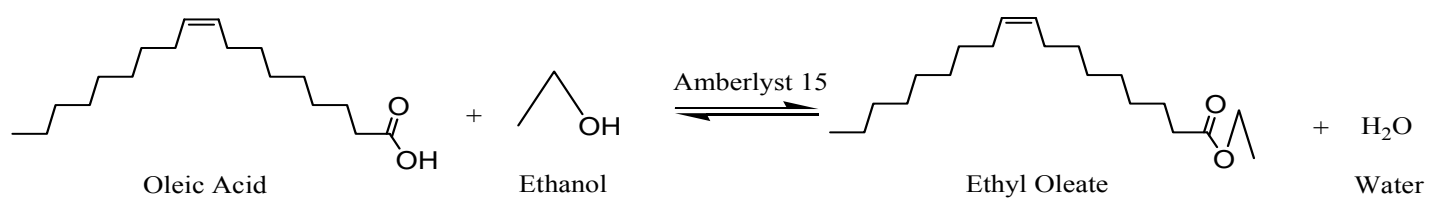

Scheme 1: Esterification of oleic acid and ethanol to ethyl oleate and water.

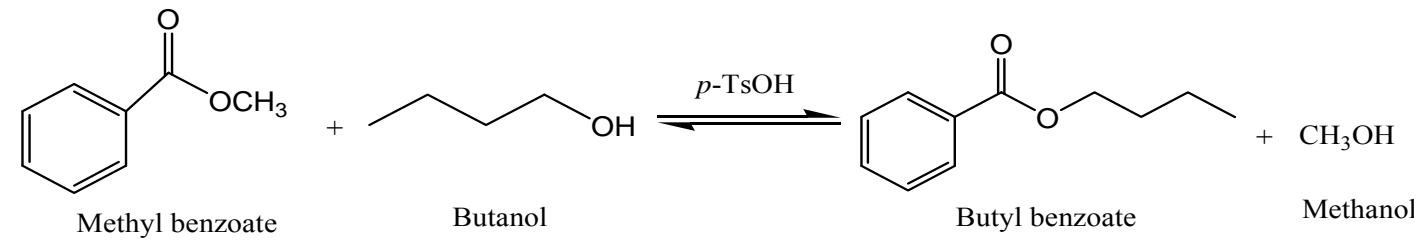

Scheme 2: Transesterification of methyl benzoate and butanol to butyl benzoate and methanol.

This paper reports on two reactions (one esterification, one transesterification) that produce water and methanol as the compounds to be selectively permeated. By tracking the performance of a single membrane system for these two reaction systems, the general applicability of the concept will be demonstrated.

\section{Materials and Methods}

2.1 Membranes and Membrane Reactor System

The membranes, CMS-7-ePTFE ${ }^{\circledR}$, used in this work were supplied by Compact Membrane Systems, Inc. (CMS). The composite membranes are made of perfluoro-2,2dimethyl-1,1,3-dioxole copolymerized with tetrafluoroethylene with varying copolymer ratios (such as AF2400 and AF1600) [28, 29] on e-PTFE support. Gas permeation tests (constant pressure/variable volume apparatus) with nitrogen, oxygen, and helium were performed on disc membranes before and after they were used for pervaporation 
experiments. Membranes were examined (cross-section and membrane surface) by Scanning Electron Microscopy (SEM) right after they were cut from a flat sheet and after they were used for pervaporation experiments. The membrane samples for SEM were prepared by sectioning with two tweezers in liquid nitrogen.

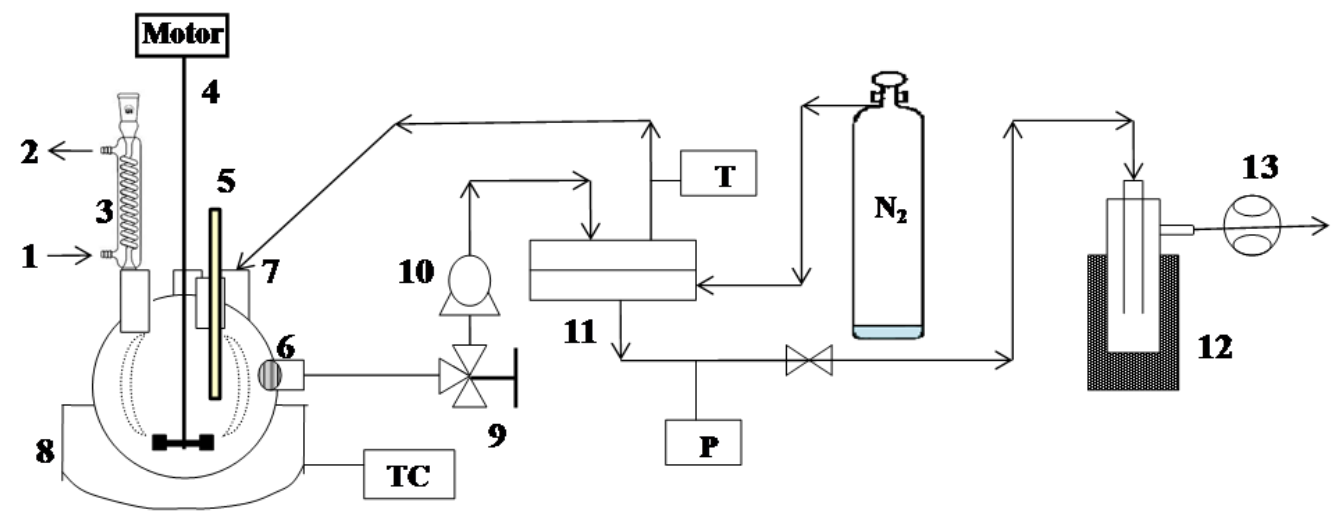

Figure 1: Schematic of the pervaporation membrane reactor system. 1,2-Cold water inlet-outlet to reflux condenser; 3-reflux condenser; 4-agitator; 5-thermocouple; 6reaction mixture outlet with an inline filter; 7-retentate line; 8-heating mantle; 9sample port; 10-circulation pump; 11-membrane; 12-liquid nitrogen cold trap; 13vacuum pump.

The pervaporation membrane reactor system is shown in Figure 1. The feed solution was kept in a $500 \mathrm{~mL}$ five-neck round-bottom glass reactor with a reflux condenser. The reactor temperature was controlled by a hemispherical mantle (Thermo Scientific). An inline filter in the reactor maintains the catalyst inside the reactor. A circulation pump (FMI lab pump Model QV, fluid metric Inc.) was used to circulate the reactant mixture 
through the membrane module. The selected membrane was installed into a modified 47 mm stainless steel filter holder (EMD Millipore), which provided a filtration area of 13.8 $\mathrm{cm}^{2}$. All components of the feed side circulation system were built using 1/8' stainless steel tubing and fittings (Swagelok, Inc.). On the permeate side, a liquid nitrogen cold trap was connected to condense the permeate, and a vacuum pump (RV5, BOC Edwards) created low pressure at the permeate side of the membrane cell (typically 0.002-0.004 bar). The permeate pressure was measured using an absolute pressure transducer (type 122A, MKS instruments). The permeate side of the membrane was purged with nitrogen (UHP/zero grade) to assist the byproduct removal.

The scale of the membrane reactor system is relatively small with only a few grams of each reactant added to the initial feed mixture. Precise amounts for each compound are provided below. For the membranes employed, run times are on the order of many hours. Thus, the average effective permeation rate for the desired alcohol (methanol or water) averages to less than 0.1 gram per hour. Using the liquid nitrogen cooled trap system employed, it was necessary to collect at least 2 grams of material to minimize error in the measurements. Thus, the permeate was aggregated over the entire run and the total mass and average composition were measured.

\subsection{Esterification and Transesterification Experiments}

For esterification reactions, oleic acid (NF/FCC grade), hexane (GC resolv grade) and ethyl oleate ( $>=98 \%$ ) were obtained from Fischer Scientific. Ethanol (EtOH, 99.5\%, anhydrous, 200 proof) was purchased from Acros Organics. The catalyst Amberlyst ${ }^{\circledR} 15$ (hydrogen form, strongly acidic, cation exchanger, dry, moisture $\sim 5 \%$ ) was purchased 
from Sigma-Aldrich. The reactor was heated to $65^{\circ} \mathrm{C}$ and was charged sequentially with oleic acid $(0.5 \mathrm{~mol})$, ethanol $(1.0 \mathrm{~mol})$ and catalyst $(5 \% \mathrm{w} / \mathrm{w})$. Once all components were charged into the reactor, aliquots $(200 \mu \mathrm{L})$ were sampled periodically from the sample port. Then, hexane ( $2 \mathrm{~mL}$ as an internal standard for gas chromatography analysis) was added to the sample and mixed by a vortex mixer. $1 \mu \mathrm{L}$ analyte was injected into a HP 6890 series gas chromatograph (FID detector, capillary column CP-Sil 88, 100 m length, $0.25 \mathrm{~mm}$ i.d., oven temperature $250^{\circ} \mathrm{C}$, column temperature $181^{\circ} \mathrm{C}$; J\&W Scientific) for analysis. During pervaporation experiments, permeate was collected in a cold trap over the entire duration of an individual experiment and was analyzed at the end of each experiment. The control experiments were carried out in the apparatus without the membrane and under the same conditions as pervaporation experiments to determine the contribution of pervaporation to the completion of the reaction.

For transesterification reactions, n-butanol $(\mathrm{BuOH}, 99 \%)$, methanol $(\mathrm{MeOH}, 99.9 \%$, HPLC grade), methyl benzoate (MeBe, reagent grade), $p$-xylene ( $>=99 \%$, certified), homogeneous catalyst $p$-toluenesulfonic acid (monohydrate, crystalline/certified) and sodium bicarbonate $(99.7+\%$, reagent ACS, powder) were purchased from Fischer

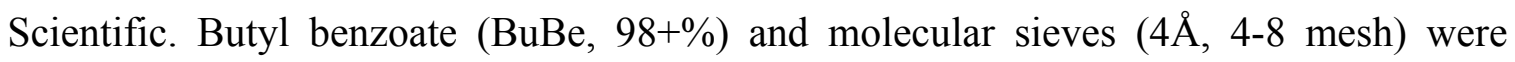
purchased from Sigma-Aldrich. To determine the thermodynamic equilibrium, both forward and reverse reactions were carried out in the batch reactor system without membranes. In forward reactions, methyl benzoate $(0.04 \mathrm{~mol})$, catalyst $p$-toluenesulfonic acid $(0.02 \mathrm{~g} / \mathrm{mmol}$ total reactants) and organic solvent $\mathrm{p}$-xylene (the molar ratio of $\mathrm{p}$ xylene/methyl benzoate $=1$ ) were charged into the reactor with a stirring speed of $200 \mathrm{rpm}$. After the mixture was heated to $75^{\circ} \mathrm{C}$, n-butanol $(0.04 \mathrm{~mol})$ was added. In reverse 
reactions, butyl benzoate $(0.04 \mathrm{~mol})$ and methanol $(0.04 \mathrm{~mol})$ were used as reactants and remains were the same as forward reactions. For pervaporation experiments in the membrane reactor system, an equimolar ratio of reactants methyl benzoate and butanol, plus p-xylene (molar ratio of p-xylene/methyl benzoate $=4$ ) or toluene (molar ratio of toluene/methyl benzoate $=3)$ and catalyst $p$-toluenesulfonic acid $(0.05 \mathrm{~g} / \mathrm{mmol}$ total reactants) were used. The reason that organic solvents were included in reaction mixtures was to evaluate CMS-7 membranes performance after exposure to aggressive solvents, as well as the membrane byproduct removal ability at low byproduct concentrations. For analysis, aliquots $(200 \mu \mathrm{L})$ were withdrawn periodically from the sample port. Then, 1 $\mathrm{mL}$ of hexane and $1 \mathrm{~mL}$ of oversaturated sodium bicarbonate solution were added to quench the reaction and neutralize the catalyst. Two phases were observed immediately. The supernatant was removed and injected into a Varian 3800 gas chromatograph equipped with a capillary column DB-WAX (30 m length, $0.25 \mathrm{~mm}$ i.d., J\&W Scientific) and a flame ionization detector (FID).

\section{Results and Discussion}

\subsection{Esterification reaction results}

Since the oleic acid is NF/FCC grade, it is a mixture of oleic acid and trace amount of other fatty acids. Therefore, the ester products are a mixture of ethyl esters. Figure 2 shows the yield of ethyl esters against the time for esterification reaction in the membrane reactor system and in the conventional system. In the conventional system, the product yield increases to $71 \%$ after 75 hours and is still slowly increasing. According to Okamoto [30], the equilibrium constant of this esterification reaction is predicted to be 
3.0 at $65^{\circ} \mathrm{C}$. Thus, the equilibrium conversion should be $81 \%$ for a $2 / 1$ molar ratio of ethanol to oleic acid reacted at $65^{\circ} \mathrm{C}$. In the membrane reactor system operating at these conditions, a relatively faster reaction rate (exceeding equilibrium after 35 hours) is observed and the product yield reached $96 \%$ with selective water removal. The GC analysis of the permeate collected at the end of the pervaporation experiment shows that no ethyl esters permeate the membrane. This result indicates that the membrane reactor is capable of selectively removing by-product water and increasing the product yield. Lucena et al. reported equivalently high ethyl ester yields of $95.5 \pm 0.7 \%$ but required higher temperatures $\left(110^{\circ} \mathrm{C}\right)$, an initial molar ratio of ethanol to oleic acid of 9 , and the use of a reactor system with an adsorption column for water removal [15]. By employing a membrane reactor system in this work, the product yield is increased by more than $15 \%$ beyond the conventional thermodynamic limit via a reactor operating at relatively mild conditions with limited excess reactants. 


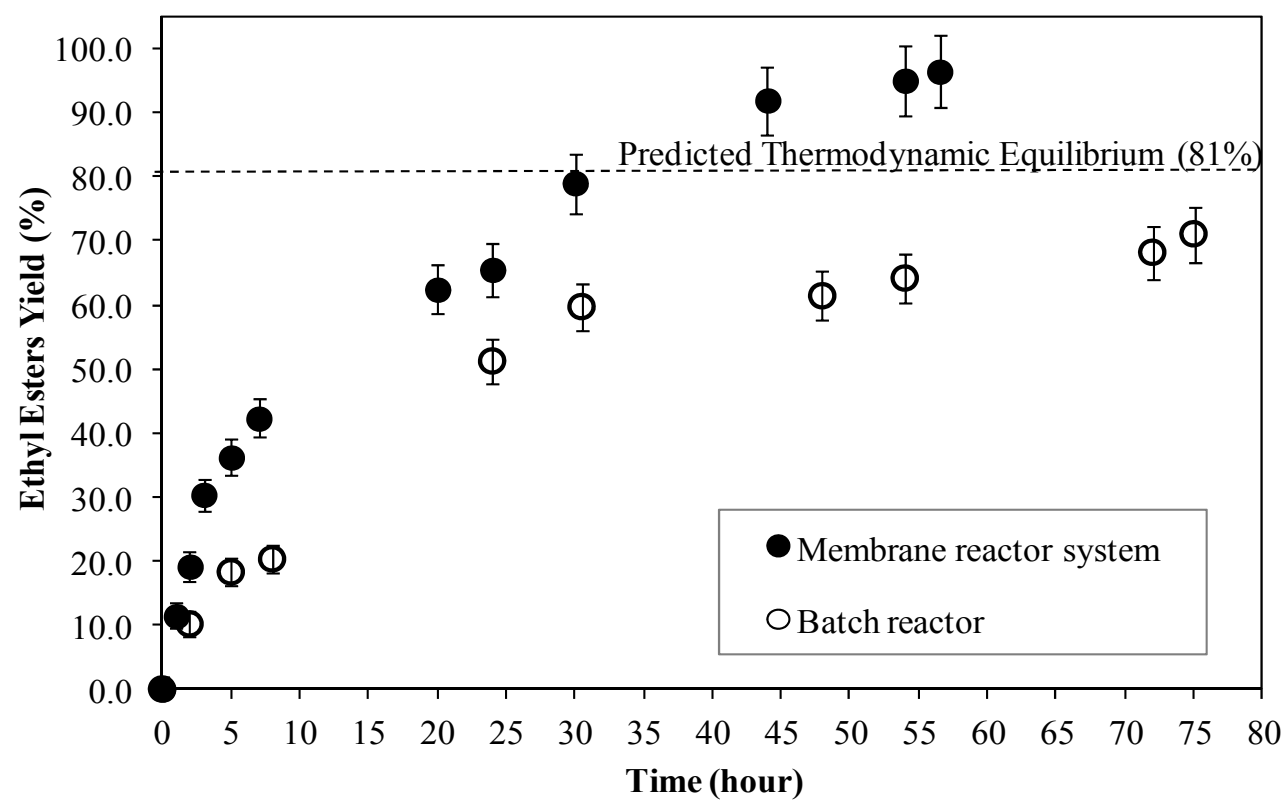

Figure 2: Esterification reaction ethyl esters yield versus time. A membrane reactor system equipped with a CMS7-ePTFE ${ }^{\circledR}$ membrane was employed. This run was made at $65^{\circ} \mathrm{C}$ and with a molar ratio of ethanol/oleic acid $=2$.

\subsection{Transesterification reaction results}

Kinetic curves of methyl benzoate conversion for both forward reaction and reverse reaction carried out in a batch reactor are shown in Figure 3. The forward reaction kinetic curve and the reverse reaction kinetic curve are almost symmetric, which means that the forward reaction rate constant should be almost equal to the reverse reaction rate constant. After 25 hours, the reaction conversion reached $52 \%$ and remained constant for the rest of the test period, which indicated that the thermodynamic equilibrium is $52 \%$ conversion when operated with an equimolar reactant ratio at $75^{\circ} \mathrm{C}$. This result also indicates that there is no side reaction happened in the reaction system. 


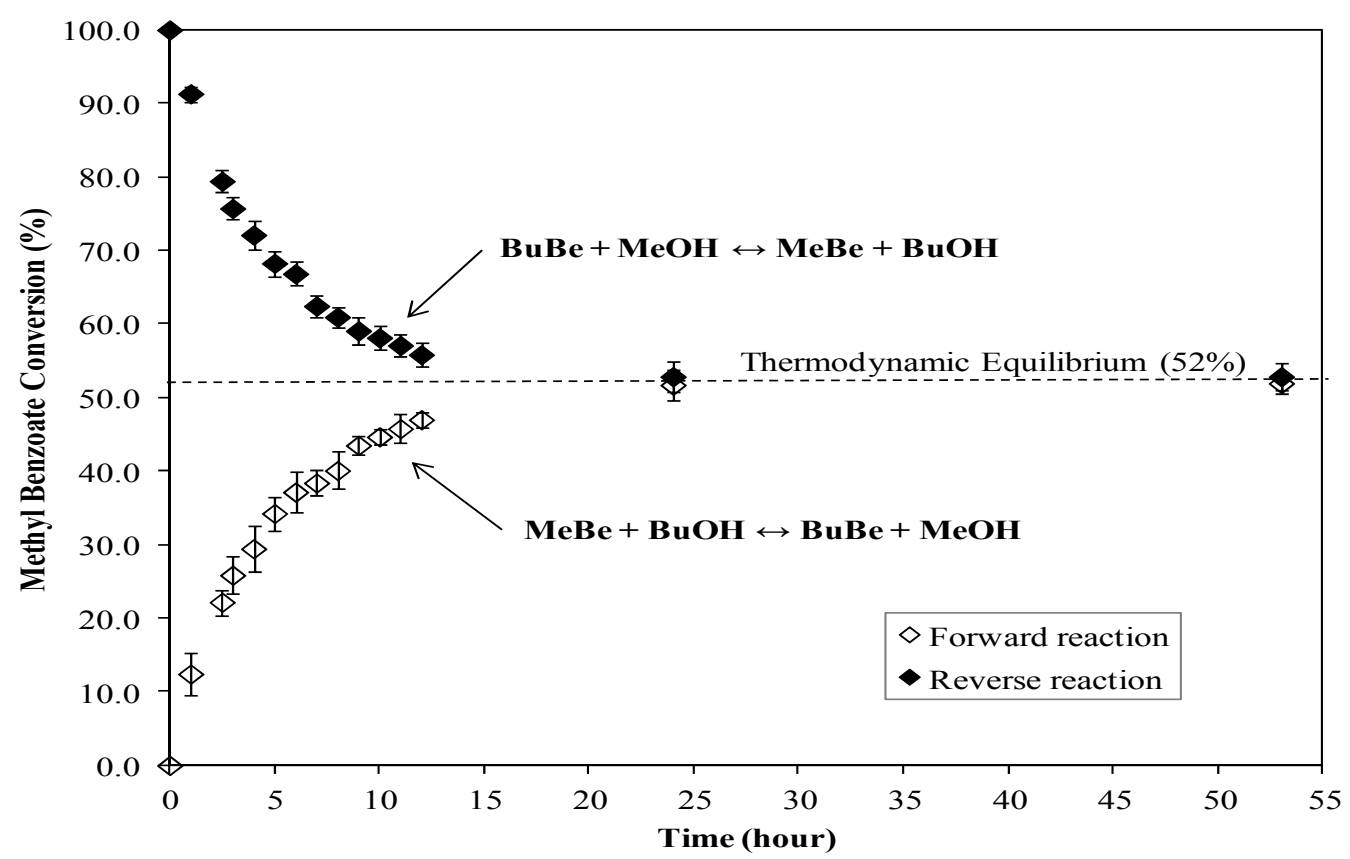

Figure 3: Transesterification forward and reverse reaction conversion versus time in a batch reactor without pervaporation. This run was made at $75^{\circ} \mathrm{C}$ and with a molar ratio of $\mathrm{MeBe} / \mathrm{BuOH} / \mathrm{p}$-xylene $=1$.

In Figure 4, a typical transesterification conversion profile in a pervaporation-assisted membrane reactor is presented, along with the corresponding profile in a conventional reactor. With the assistance of pervaporation, the system rapidly reached $77 \%$ conversion, which exceeds the thermodynamic equilibrium by $25 \%$. Compared to the batch reaction without pervaporation, a relatively fast reaction rate (nearly $20 \%$ conversion within the first hour) and a short reaction time (5 hours) prior to exceeding equilibrium were also achieved in the membrane reactor system. Analysis of the permeate collected during the membrane-assisted reaction indicates that the permeate consists of $88.7 \mathrm{~mol} \%$ methanol, $1.2 \mathrm{~mol} \%$ butanol and $10.1 \mathrm{~mol} \% \mathrm{p}$-xylene. Therefore, the membrane reactor is capable of selectively removing methanol and shifting the equilibrium towards higher conversion. 


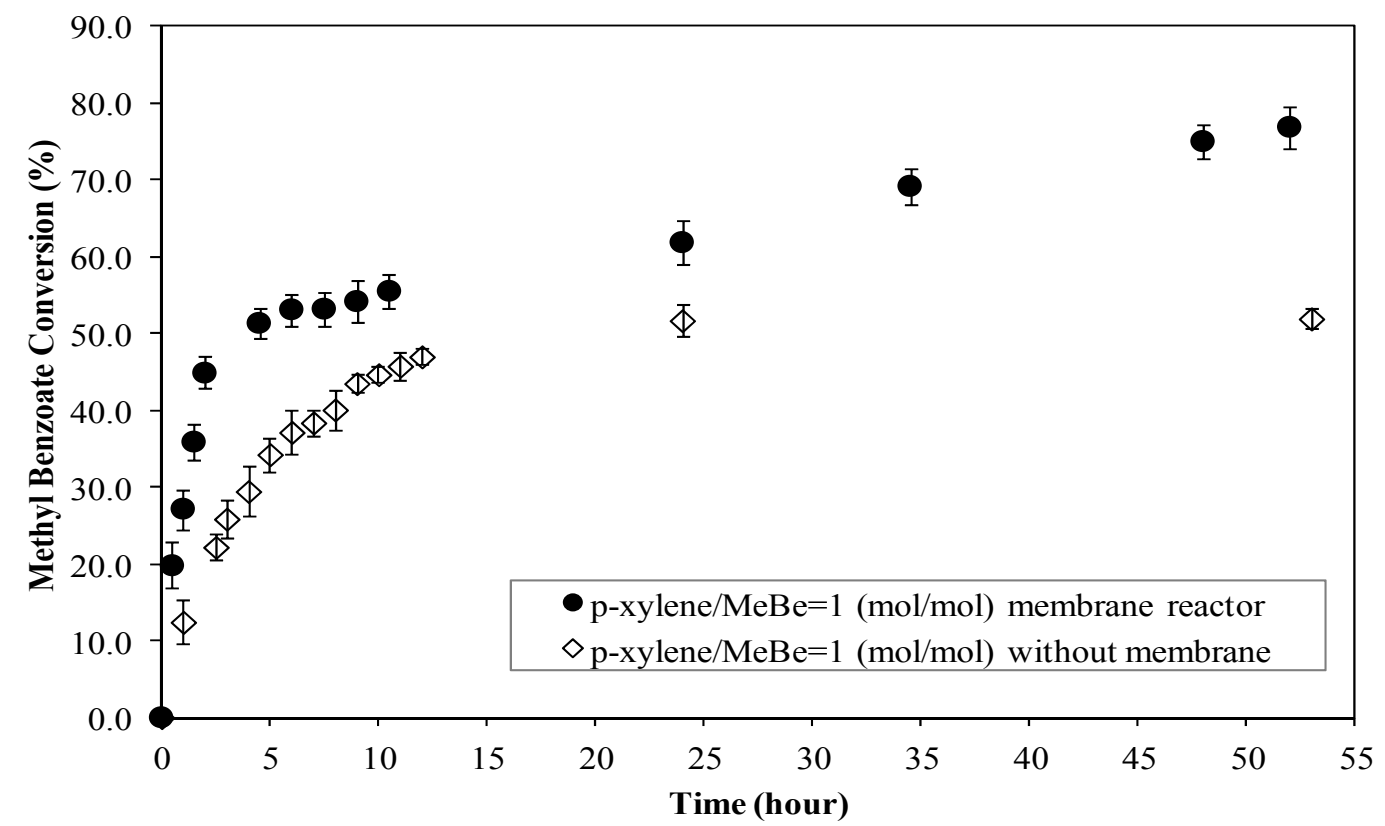

Figure 4: Transesterification reaction conversion versus time. A membrane-assisted reactor equipped with a CMS7-ePTFE ${ }^{\circledR}$ membrane was employed. This run was made at $75^{\circ} \mathrm{C}$ and with a molar ratio of $\mathrm{MeBe} / \mathrm{BuOH} / \mathrm{p}$-xylene $=1$.

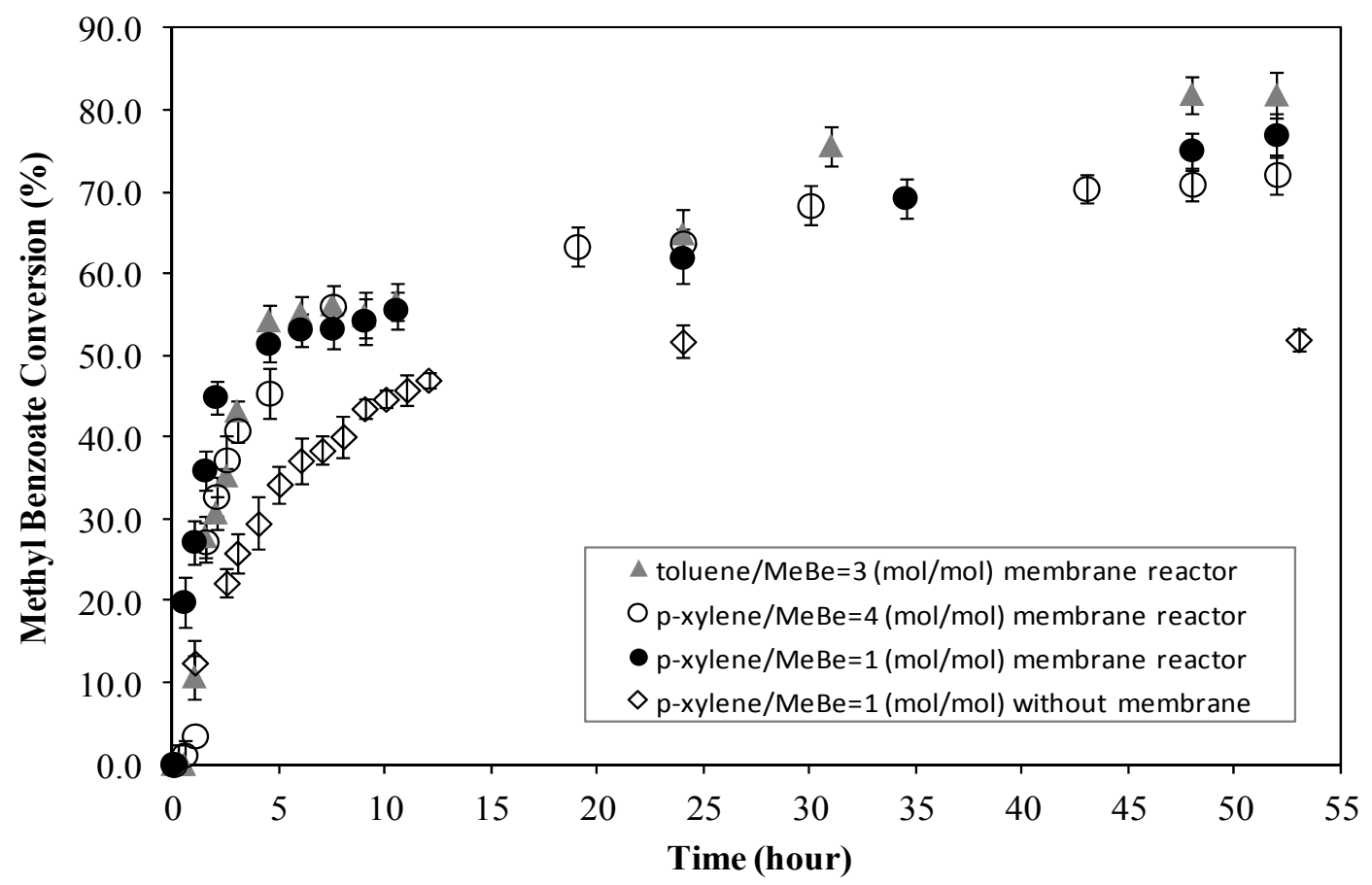

Figure 5: Process dynamics for transesterification reaction with differnet solvent. All runs were carried out at $75^{\circ} \mathrm{C}$ and with a catalyst concentration of $0.05 \mathrm{~g}$ catalyst /mmol total reactants. 
Figure 5 compares the conversions of transesterification reaction in different solvent medium in a membrane reactor system. The lower curve presents the concentration of product, methyl benzoate, as a function of reaction time for the conventional system. The upper curves present the same function for three membrane reactor runs operated with varying amounts of pyridine solvent in the system. Analysis of the curves indicates that the reaction rate is very rapid during the first five hours of reaction and then steadily decreases, consistent with the behavior expected from a reaction with product inhibition. The reaction rates and conversion achieved in the membrane reactor system were in all cases distinctly higher than those achieved in a conventional reactor. Additionally, all membrane reactor cases follow the same trend, rapid increase in product over the first five hours followed by a slower increase from five to 55 hours. The final conversion achieved in each of the membrane reactor systems was $75+/-5 \%$. Thus, the ability of methanol removal by the CMS-7 membrane is almost unaffected by the methanol concentration. For the three conditions studied, as the ratio of solvent to methyl benzoate in the reactor increased, the concentration of the produced methanol decreased. Since the conversion versus time curves for these three conditions are nearly identical, the permeation rate of methanol from the reactor appears to be nearly independent of concentration over the range of conditions studies.

Bazi et al. carried out the same transesterification reaction in a batch reactor at $110^{\circ} \mathrm{C}$ and obtained $100 \%$ conversion after 48 hours of reaction time using a molar ratio of butanol/MeBe of $11 / 2$ and in toluene medium [31]. Clearly, by incorporating CMS-7 membranes, which are capable of selectively removing methanol, the transesterification reaction can be operated at a lower temperature and no excess alcohol is required. 
Table 1: The impact of amount of solvent and solvent type on the permeate composition of the pervaporation assisted transesterification of methyl benzoate with butanol at $75^{\circ} \mathrm{C}$ and equimolar reactant addition. The permeate was analyzed at the end of each run (after around 52 hours).

\begin{tabular}{|c|c|c|c|c|c|c|}
\hline & $\begin{array}{c}\text { Solvent to } \\
\text { Reactant Ratio } \\
(\mathbf{m o l} / \mathbf{m o l})\end{array}$ & $\begin{array}{c}\text { MeOH } \\
\text { Capture }^{\mathrm{a}} \\
(\%)\end{array}$ & $\begin{array}{c}\text { MeOH } \\
\text { Removal }^{\mathrm{b}} \\
(\%)\end{array}$ & $\begin{array}{c}\text { Reaction } \\
\text { Conversion } \\
(\%)\end{array}$ & $\begin{array}{c}\text { Max } \\
\text { Conversion }^{\mathrm{c}} \\
(\%)\end{array}$ & $\begin{array}{c}\mathrm{MeOH} / \mathrm{BuOH} \\
(\mathrm{mol} / \mathrm{mol}) \text { in } \\
\text { permeate }\end{array}$ \\
\hline 1 & p-xylene $/ \mathrm{MeBe}=1$ & 76 & 89 & 77 & 99 & 72 \\
\hline 2 & p-xylene $/ \mathrm{MeBe}=4$ & 75 & 82 & 72 & 97 & 18 \\
\hline 3 & Toluene $/ \mathrm{MeBe}=3$ & 91 & 94 & 82 & 91 & 9 \\
\hline
\end{tabular}

a. $\mathrm{MeOH}$ Capture $(\%)=$ measured $\mathrm{mmol}$ of $\mathrm{MeOH}$ in trap $/(\mathrm{mmol} \mathrm{MeBe}$ fed $\times$ reaction conversion $) \times 100 \%$

b. $\mathrm{MeOH}$ Removal $(\%)=$ calculated $\mathrm{mmol}$ of $\mathrm{MeOH}$ needed to be removed to achieve reaction conversion $/(\mathrm{mmol} \mathrm{MeBe}$ fed $\times$ reaction conversion $) \times 100 \%$

c. Max Conversion $(\%)=(\mathrm{mmol} \mathrm{MeBe}$ fed $-\mathrm{mmol}$ of $\mathrm{BuOH}$ in trap $) / \mathrm{mmol} \mathrm{MeBe}$ fed $\times 100 \%$

Table 1 compares the percentage of methanol captured in cold traps with the calculated percentage of methanol removal to achieve the measured conversion. The solvent type and the amount of solvent were varied in each run for this comparison. The evaluated permeate molar compositions were cumulative over the entire reaction period. The $\mathrm{MeOH}$ capture is the percentage of the methanol produced in the reaction, as determined from the methyl benzoate conversion and assuming no side reactions, that was recovered in the trap. The methanol capture for these three runs is in the range of $75 \%-91 \%$. Therefore, the residual methanol in the reaction mixture ranged from 9 to $25 \%$ of the methanol formed.

The permeate of all pervaporation assisted transesterification reaction runs was primarily methanol with lesser amounts of butanol and p-xylene or toluene and no esters. If we assume that no butanol permeates the membrane and that equilibrium is achieved in the reactor, the moles of methanol produced can be calculated from the equilibrium constant of this transesterification reaction $\left(\mathrm{K}_{\mathrm{eq}}=1.17\right)$ and the initial esters and butanol concentrations. Therefore, the $\mathrm{MeOH}$ removal can be defined as the percentage of the methanol that was produced that would have to be removed from the reactor to achieve 
the measured conversion. From table 1 , the methanol removal is in the range of $82 \%-94 \%$. This range matches reasonably well with that of the methanol capture. In all cases, the methanol capture is slightly less than the methanol removal indicated that there may have been a very small loss of methanol vapor through the cold trap.

As the concentration of $\mathrm{p}$-xylene increased from run 1 to run 2, more $\mathrm{p}$-xylene might sorb in the selective layer and swell the membrane, which could cause the observed decrease of membrane selectivity of methanol over butanol. Compared to $\mathrm{p}$-xylene, toluene has higher potential swelling capability, as evidenced by their Hansen solubility parameter $(\delta$ of toluene $=18.2 \mathrm{MPa}^{0.5}$ and $\delta$ of $\mathrm{p}$-xylene $\left.=17.9 \mathrm{MPa}^{0.5}[32]\right)$. Therefore, toluene is more likely to sorb in the selective layer and to swell the membrane more than p-xylene, which would cause the membrane selectivity of $\mathrm{MeOH} / \mathrm{BuOH}$ in run 3 to be lower than that in run 2 and run 1 . This is supported by the fact that the permeate contained a higher molar ratio of toluene than $p$-xylene even if less amount of $p$-xylene was feed into the reactor at the beginning. Additionally, even though the methanol to butanol selectivity for all systems was quite high, it was the lowest for the run in which toluene was used as the solvent.

Using the experimentally measured reactor performance and permeation properties, we developed a mathematical model that predicts reactor performance based on membrane permeation rates and permselectivities. The details of this model are reported in a related publication [26].

\subsection{Pervaporation membranes}

Figure 6A-E shows the SEM images of the entire CMS-7 ePTFE ${ }^{\circledR}$ membrane, which includes selective layer (before and after used for pervaporation) and porous support. 
From these images, the thickness of a new CMS-7 $\mathrm{ePTFE}^{\circledR}$ membrane can be estimated to be about $42 \mu \mathrm{m}$ while the dense layer has an approximate thickness of $3.2 \mu \mathrm{m}$ and the porous layer is about $38.8 \mu \mathrm{m}$. Figure $6 \mathrm{D}$ is an image of the back side of a used membrane. Since the membrane was sitting on top of the support screen inside the membrane holder during the pervaporation experiments, there are protrusions formed on the back side of the used membrane. It can be seen from Figure 6E that the whole thickness of used membranes is thinner than that of unused membranes. The selective layer of a used membrane has a thickness of about $1.8 \mu \mathrm{m}$ and the porous support layer is about $10 \mu \mathrm{m}$. The decrease in thickness is due to the compression caused by the pressure difference on both sides of the membrane during the pervaporation process. It is also noticed that at some locations of membranes there is a separation between the dense layer and the porous layer after membranes are used in pervaporation experiments.
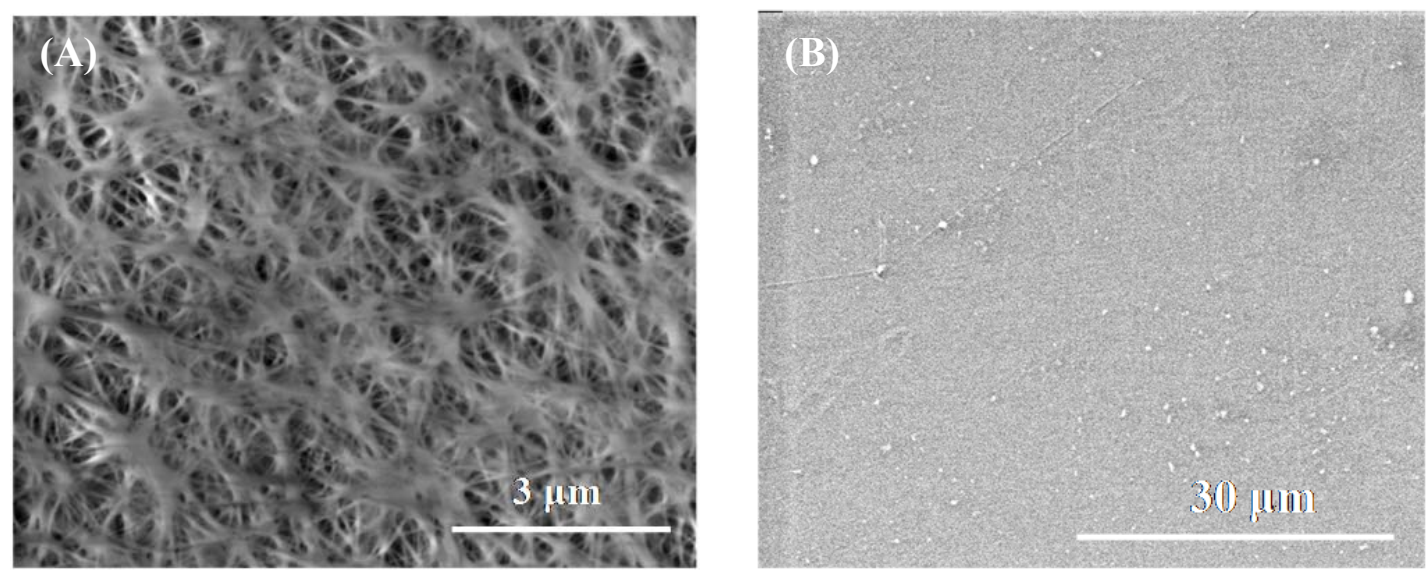


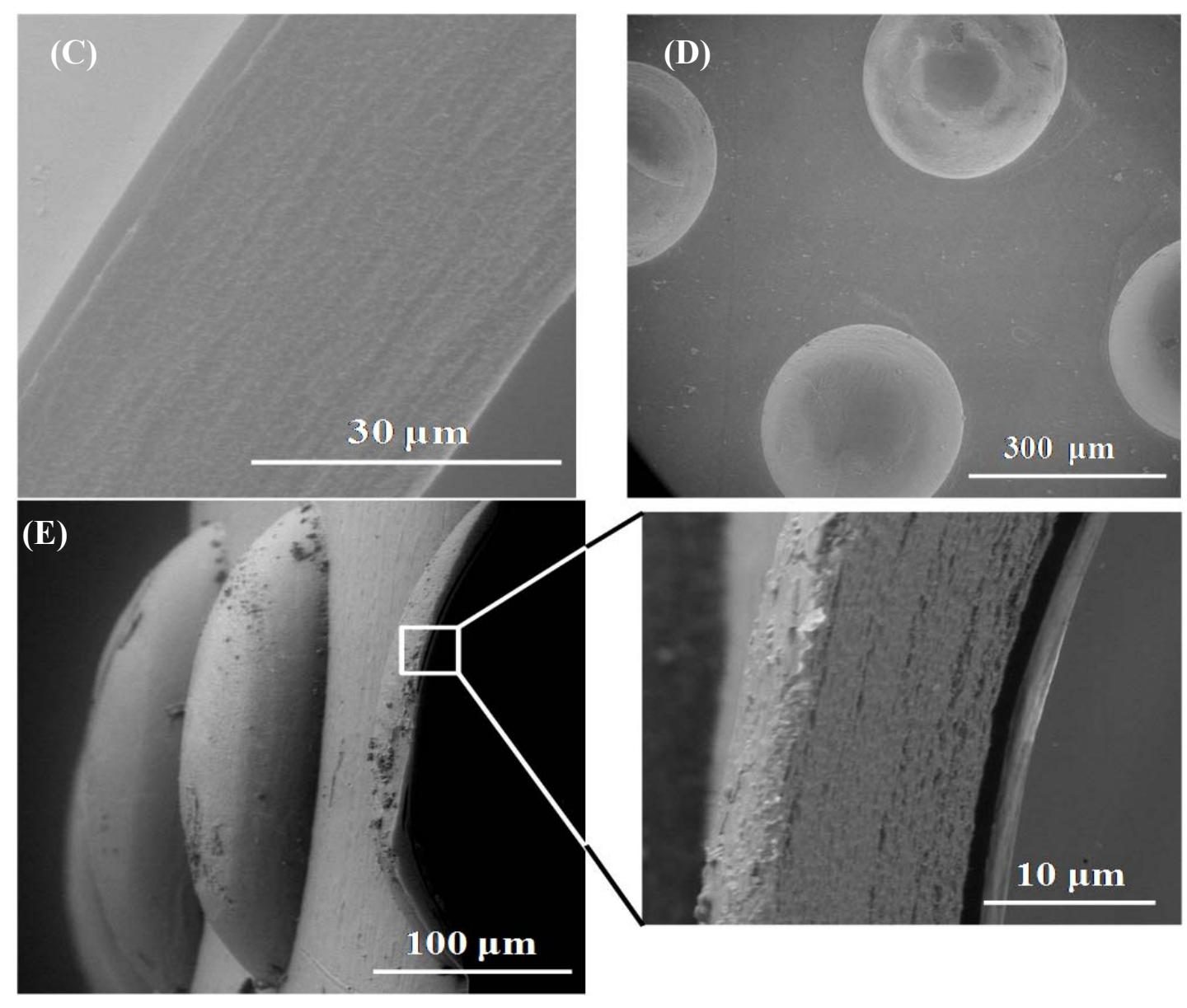

Figure 6: SEM images: (A) back side of an unused membrane (e-PTFE porous support) (B) denser layer of an unused membrane (C) cross-section of an unused membrane (D) back side of a used membrane (E) cross-section of a used membrane.

A series of 10 pervaporation experiments was carried out in a membrane reactor system equipped with a CMS-7 ePTFE $^{\circledR}$ membrane. During a discrete 400 hours of reaction time, a single membrane stamp was kept in a temperature range of $20-75^{\circ} \mathrm{C}$. The chemicals and solvents that contacted with membrane include methanol, butanol, ethanol, water, pxylene, toluene, oleic acid, strong homogeneous acid catalyst p-toluenesulfonic acid, methyl benzoate and butyl benzoate. The measured gas selectivities for this CMS-7 $\mathrm{ePTFE}^{\circledR}$ membrane employed in all pervaporation experiments are listed in table 2. From the data, we can observe that there are only small decreases in membrane selectivities and permeance after it was used for pervaporation-assisted esterification reactions and 
transesterification reactions. Therefore, we can conclude that CMS-7 ePTFE ${ }^{\circledR}$ membranes have excellent long-term stability as well as thermal and chemical resistance when they are used in the temperature range of room temperature to $75^{\circ} \mathrm{C}$ in the presence of organic solvents and strong homogeneous acid catalysts.

We have employed gas permeation measurements as a test of the integrity of the membranes employed because such measurements are both highly accurate and facile to complete. They give quite useful information about subtle changes in the porous membrane substructure as well as the selective skin. As can be seen from evaluation of the before and after use data reported in table 2, the membranes employed here experienced a reduction in permeance of about $15 \%$ for most gases. Interestingly, the nitrogen flux remained constant over the course of these runs. These permeation losses might be attributable to either modest compaction of the support or to a loss in permeability of the skin layer. Based on the information available at this time, it is not possible to unequivocally identify the source of change.

Table 2: CMS-7 ePTFE $^{\circledR}$ membrane gas permeance and selectivity measured in a constant pressure/variable volume apparatus.

\begin{tabular}{ccccc}
\hline \multirow{2}{*}{ Gas } & \multicolumn{2}{c}{ Permeance $\left(\mathbf{G P U}^{*}\right)$} & \multicolumn{2}{c}{ Selectivity $\left(\right.$ over $\left.\mathbf{N}_{2}\right)$} \\
Before & After & Before & After \\
\hline $\mathbf{N}_{\mathbf{2}}$ & 117 & 117 & - & - \\
$\mathbf{O}_{\mathbf{2}}$ & 241 & 204 & 2.1 & 1.8 \\
$\mathbf{H}_{\mathbf{2}}$ & 525 & 444 & 4.5 & 3.8 \\
$\mathbf{H e}$ & 579 & 501 & 5.0 & 4.3 \\
\hline $1 \mathrm{GPU}=1 \times 10^{-6} \mathrm{~cm}^{3}(\mathrm{STP}) / \mathrm{cm}^{2} \cdot \mathrm{s} \cdot \mathrm{cmHg}$ & &
\end{tabular}




\section{Conclusions}

A continuous pervaporation-assisted membrane reactor system was developed with CMS7-ePTFE ${ }^{\circledR}$ membranes. Esterification reaction of oleic acid with ethanol and transesterification reaction of methyl benzoate with butanol were studied as two model reactions for organic synthesis in this membrane reactor system. In both cases, a relatively fast reaction rate and a markedly high conversion were achieved in the membrane reactor system than those achieved in batch reactor. CMS7-ePTFE ${ }^{\circledR}$ membranes have high selectivity of byproduct (water or methanol) over reactants and ester products. The loss of esters and reactant alcohols into permeate during reactions carried out in the membrane reactor system can be neglected under experiment conditions in this work. By selective removal of a by-product (water or methanol), the reaction conversion was promoted by $15 \%$ for esterification reaction and $25 \%$ for transesterification reaction. To achieve the same or even higher degree of conversion than that reached in a conventional reactor, the pervaporation-assisted membrane reactor system equipped with CMS7-ePTFE ${ }^{\circledR}$ membranes can be operated at lower temperature and consume much less amount of alcohol reactant. Good stability and chemical resistance of these membranes have been proved by no significant loss of gas permeance and selectivity after all pervaporation experiments. This membrane reactor system can be employed to improve chemical synthesis process in terms of operation conditions and reactant consumption, and it is especially suitable for reactions involving temperature and $\mathrm{pH}$ sensitive substances. 


\section{Acknowledgements}

This project is supported by the Department of Energy (Grant No. DE-FG02-07ER86305)

and the SBIR program (Grant No. DE-FG02-06ER84529).

\section{Reference}

[1] Mulder, M. (1996) Basic Principles of Membrane Technology, 2nd ed., Springer.

[2] Baker, R. (2004) Membrane Technology and Applications, 2nd ed., John Wiley \& Sons, Ltd., England.

[3] Ho, W.; Sirkar, K. (1992) Membrane Handbook, 1 ed., Springer.

[4] Barahona, D.; Pfromm, P.; Rezac, M. (2006) Effect of water activity on the lipase catalyzed esterification of geraniol in ionic liquid [bmim]PF6, Biotechnol. Bioeng., 93: 318.

[5] Waldburger, R.; Widmer, F. (1996) Membrane reactors in chemical production processes and the application to the pervaporation-assisted esterification, Chem. Eng. Technol., 19: 117.

[6] Sirkar, K.; Shanbhag, P.; Kovvali, A. (1999) Membrane in a reactor: A functional perspective, Ind. Eng. Chem. Res., 38: 3715.

[7] Castanheiro, J.; Ramos, A.; Fonseca, I.; Vital, J.(2006) Esterification of acetic acid by isoamylic alcohol over catalytic membranes of poly(vinyl alcohol) containing sulfonic acid groups, Appl. Catal., A, 311: 17.

[8] Hasanoglu, A.; Salt, Y.; Keleser, S.; Dincer, S. (2009) The esterification of acetic acid with ethanol in a pervaporation membrane reactor, Desalination, 245: 662.

[9] Tanaka, K.; Yoshikawa, R.; Ying, C.; Kita, H.; Okamoto, K. (2002) Application of zeolite $\mathrm{T}$ membrane to vapor-permeation-aided esterification of lactic acid with ethanol, Chem. Eng. Sci., 57: 1577.

[10] P.G. Cao, M.A. Dube, A.Y. Tremblay, (2008) High-purity fatty acid methyl ester production from canola, soybean, palm, and yellow grease lipids by means of a membrane reactor, Biomass Bioenerg, 32: 1028.

[11] Bartling, K.; Thompson, J.; Pfromm, P.; Czermak, P.; Rezac, M. (2001) Lipasecatalyzed synthesis of geranyl acetate in n-hexane with membrane-mediated water removal, Biotechnol. Bioeng., 75: 676.

[12] de la Iglesia, O.; Mallada, R.; Menendez, M.; Coronas, J. (2007) Continuous zeolite membrane reactor for esterification of ethanol and acetic acid, Chem. Eng. J., 131: 35.

[13] Korkmaz, S.; Salt, Y.; Dincer, S. (2011) Esterification of Acetic Acid and Isobutanol in a Pervaporation Membrane Reactor Using Different Membranes, Ind. Eng. Chem. Res., 50: 11657. 
[14] Jafar, J.; Budd, P.; Hughes, R. (2002) Enhancement of esterification reaction yield using zeolite - A vapour permeation membrane, J. Membr. Sci., 199: 117.

[15] Lucena, I.; Saboya, R.; Oliveira, J.; Rodrigues, M.; Torres, A.; Cavalcante, C.;. Parente, E.; Silva, G.; Fernandes, F. (2011) Oleic acid esterification with ethanol under continuous water removal conditions, Fuel, 90: 902.

[16] Hanh, H.; Nguyen, T.; Okitsu, K.; Nishimura, R.; Maeda, Y. (2009) Biodiesel production by esterification of oleic acid with short-chain alcohols under ultrasonic irradiation condition, Renewable Energy, 34: 780.

[17] Lucena, I.; Silva, G.; Fernandes, F. (2008) Biodiesel production by esterification of oleic acid with methanol using a water adsorption apparatus, Ind. Eng. Chem. Res., 47: 6885.

[18] Oliveira, A.; Rosa, M; Aires-Barros, M.; Cabral, J. (2001) Enzymatic esterification of ethanol and oleic acid - a kinetic study, J. Mol. Catal. B: Enzym., 11: 999.

[19] Cardoso, A.; Neves, S.; da Silva, M. (2008) Esterification of Oleic Acid for Biodiesel Production Catalyzed by $\mathrm{SnCl}(2)$ : A Kinetic Investigation, Energies, 1: 79.

[20] Van Gerpen, J. (2005) Biodiesel processing and production, Fuel Process. Technol., 86: 1097-1107.

[21] Ma, F.; Clements, L.; Hanna, M. (1998) The effects of catalyst, free fatty acids, and water on transesterification of beef tallow, Trans. ASAE, 41: 1261.

[22] Tesser, R.; Di Serio, M.; Guida, M.; Nastasi, M.; Santacesaria, E. (2005) Kinetics of oleic acid esterification with methanol in the presence of triglycerides, Ind. Eng. Chem. Res. 44: 7978.

[23] Otera, J. (1993) Transesterification, Chem. Rev., 93: 1449.

[24] de los Rios, A.; Hernandez-Fernandez, F.; Presa, H.; Gomez, D.; Villora, G. (2009) Tailoring supported ionic liquid membranes for the selective separation of transesterification reaction compounds, J. Membr. Sci., 328: 81.

[25] Tremblay, A.; Cao, P.; Dube, M. (2008) Biodiesel production using ultralow catalyst concentrations, Energy \& Fuels, 22: 2748.

[26] Zhang, F.; Bliem, V.; Rezac, M.; Kosaraju, P.; Nemser, S. (2013) Pervaporation membrane reactors for reversible organic reactions: modeling of the membranereactor performance based on membrane properties and operating conditions, Submitted to Ind. Eng. Chem. Res.

[27] Fraile, J.; Mallada, R.; Mayoral, J.; Menendez, M.; Roldan, L. (2010) Shift of Multiple Incompatible Equilibriums by a Combination of Heterogeneous Catalysis and Membranes, Chem. Eur. J., 16: 3296.

[28] Alentiev, A.; Yampolskii, Y.; Shantarovich, V.; Nemser, S.; Plate, N. (1997)High transport parameters and free volume of perfluorodioxole copolymers, J. Membr. Sci., 126: 123.

[29] Smuleac, V.; Wu, J.; Nemser, S.; Majumdar, S.; Bhattacharyya, D. (2010) Novel perfluorinated polymer-based pervaporation membranes for the separation of solvent/water mixtures, J. Membr. Sci., 352: 41.

[30] Okamoto, K.; Yamamoto, M.; Otoshi, Y.; Semoto, M.; Yano, M.; Tanaka, K.; Kita, T. (1993) Pervaporation-Aided Esterification of Oleic-Acid, J. Chem. Eng. Jpn., 26: 475-481. 
[31] Bazi, F.; El Badaoui, E.; Sokori, S.; Tamani, S.; Hamza, M;. Boulaajaj, S. Sebti, S. (2006) Transesterification of methylbenzoate with alcohols catalyzed by natural phosphate, Synth. Commun., 36: 1585.

[32] Hansen,C.M. (2007) Hansen Solubility Parameters: A User's Handbook, 2 ed., CRC Press, Boca Raton, FL. 


\section{Captions}

Scheme 1: Esterification of oleic acid and ethanol to ethyl oleate and water.

Scheme 2: Transesterification of methyl benzoate and butanol to butyl benzoate and methanol. 
Figure 1: Schematic of the pervaporation membrane reactor system. 1,2-Cold water inlet-outlet to reflux condenser; 3-reflux condenser; 4-agitator; 5-thermocouple; 6reaction mixture outlet with an inline filter; 7-retentate line; 8-heating mantle; 9-sample port; 10-circulation pump; 11-membrane; 12-liquid nitrogen cold trap; 13-vacuum pump.

Figure 2: Esterification reaction ethyl esters yield versus time. A membrane reactor system equipped with a CMS7-ePTFE ${ }^{\circledR}$ membrane was employed. This run was made at $65^{\circ} \mathrm{C}$ and with a molar ratio of ethanol/oleic acid $=2$.

Figure 3: Transesterification forward and reverse reaction conversion versus time in a batch reactor without pervaporation. This run was made at $75^{\circ} \mathrm{C}$ and with a molar ratio of $\mathrm{MeBe} / \mathrm{BuOH} / \mathrm{p}$-xylene $=1$.

Figure 4: Transesterification reaction conversion versus time. A membrane-assisted reactor equipped with a CMS7-ePTFE ${ }^{\circledR}$ membrane was employed. This run was made at $75^{\circ} \mathrm{C}$ and with a molar ratio of $\mathrm{MeBe} / \mathrm{BuOH} / \mathrm{p}$-xylene $=1$.

Figure 5: Process dynamics for transesterification reaction with differnet solvent. All runs were carried out at $75^{\circ} \mathrm{C}$ and with a catalyst concentration of $0.05 \mathrm{~g}$ catalyst $/ \mathrm{mmol}$ total reactants.

Figure 6: SEM images: (A) back side of an unused membrane (e-PTFE porous support) (B) denser layer of an unused membrane (C) cross-section of an unused membrane (D) back side of a used membrane (E) cross-section of a used membrane. 
Table 1: The impact of amount of solvent and solvent type on the permeate composition of the pervaporation assisted transesterification of methyl benzoate with butanol at $75^{\circ} \mathrm{C}$ and equimolar reactant addition. The permeate was analyzed at the end of each run (after around 52 hours).

Table 2: CMS-7 ePTFE $^{\circledR}$ membrane gas permeance and selectivity measured in a constant pressure/variable volume apparatus. 